\title{
How Does MRI Work? An Introduction to the Physics and Function of Magnetic Resonance Imaging
}

D. Weishaupt, V.D. Köchli, and B. Marincek, eds.

New York, NY: Springer, 2006, 169 pages, \$39.95

This soft-cover booklet is a completely revised English edition published only $2 \mathrm{y}$ after the first English edition. The initial edition was met with great approval by the Englishspeaking world, providing an enormous incentive for the authors to further improve and update the text.

All chapters have been thoroughly revised and updated to include the latest developments in the ever-changing field of MRI technology. In particular, the chapter on cardiovascular imaging has been improved and expanded. Moreover, 2 completely new chapters have been added: "Fat Suppression Technique" and "High-Field Clinical MR Imaging."

This booklet, divided into 15 chapters, introduces the physics and function of MRI and presents all the important and clinically relevant aspects in a clearly structured manner. Practical information is emphasized, including the latest trends and developments relevant to clinical MRI.

The opening chapters describe the underlying physical principles of spin and MRI phenomena, relaxation, image contrast, slice selection and spatial encoding, factors affecting signal-to-noise ratio, MRI scanners, and the basic and fast-pulse sequences and fat-suppression techniques commonly used in clinical MRI. Other chapters are dedicated to more advanced techniques such as parallel imaging and cardiovascular MRI. The book is rounded out by chapters on MRI contrast agents, artifacts, high-field imaging, and bioeffects, as well as safety concerns.

The 57 figures are clearly illustrated for easier understanding of MRI physical principles and techniques. There are also 9 tables concisely summarizing image contrast, signal intensities, parameters for signal-to-noise ratio and scanning time, and contrast agents. An extensive glossary offers rapid access to the most important MRI terminology.

This book is intended for medical students, imaging residents, and technologists - in brief, all readers who, at the beginning of their MRI training, are looking for an easy-to-understand and concise introduction to this fascinating yet somewhat complex imaging modality.

The book will be helpful not to MRI specialists or MRI physicists but to nuclear medicine physicians or technologists seeking a basic understanding of MRI to apply to correlative or fused imaging with PET or SPECT.

E. Edmund Kim University of Texas M.D. Anderson Cancer Center Houston, Texas

DOI: 10.2967/jnumed.107.045104 